\title{
Next Generation Service Delivery Platforms and Service Overlay Networks
}

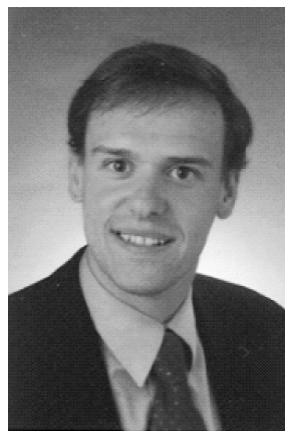

\section{Wolfgang Kellerer}

DOCOMO Comunications Laboratories Europe $\mathrm{GmbH}$

Landsberger Str. 312

80687 München

Germany

kellerer@docomolab-euro.com

Dr.-Ing. Wolfgang Kellerer is Director of the Ubiquitous Networking Research Group at DOCOMO Euro-Labs. His research includes mobile network and service platform architectures, peer-topeer overlay networks, signaling protocols and multimedia communication. He holds a Dipl.-Ing. Univ. degree in EE and IT and a Dr.-Ing. (PhD) degree from the Munich University of Technology (TUM), Germany. He is an elected member of the Steering Board of the GI/ITG Technical Committee on Communication and Distributed Systems (KuVS).

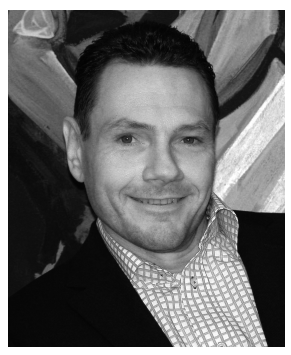

\section{Thomas Magedanz}

Technische Universität Berlin FR 5-14

Franklinstr. 28/29

10587 Berlin

Germany

tm@cs.tu-berlin.de

Prof. Dr. Thomas Magedanz is professor in the electrical engineering and computer sciences faculty at the Technische Univerität Berlin and additionally leading the competence center Next Generation Network Infrastructures (NGNI) at the Fraunhofer Institute FOKUS. His research and development activities concentrate on multimedia service delivery platform design, prototyping and optimization on top of converging fixed and mobile telecommunication networks and the Internet, including Next Generation Networks and Next Generation Mobile Networks. He is member of the GI/ITG and IEEE.

In the history of communication and information systems, specific networks have featured specific service architectures such as with voice telephony or television. With the emergence of new network technologies and in particular with the adoption of Internet (namely IP based) technologies for telecommunication and media networks, the need for a unified service infrastructure has been raised, in order to provide value-added services seamlessly on top of these networks. Such service infrastructure is commonly referred to as Service Delivery Platform (SDP).
In order to support service creation and delivery more efficiently, reusable service building blocks have been introduced within SDPs. Following such principle, Intelligent Networks (IN) have been considered as the most important SDP architecture for telecommunications in the nineties, which have been standardized by ITU-T and ETSI for fixed and mobile circuit-switched networks.

In view of the convergence of telecommunications, Internet, IT, and entertainment and the resulting diversification of value chains, SDPs for the provisioning of converged multimedia services have become increasingly complex. Today, the area of SDP concepts and systems is highly fragmented and no unified approach for converged service delivery exists.

Symptomatic for the diversity of the SDP concepts and systems deployed is that no single global SDP standardization body does exist. Whereas in the fixed and mobile telecommunications domain the IP Multimedia System (IMS) has become the defacto standard for advanced service control in a centralized way in IP-based networks, in the Internet, proprietary over the top (OTT) architectures and Peer-to-Peer (P2P) architectures promote a more distributed way of service Delivery. In addition, the notion of Service Oriented Architecture (SOA) and emerging open Application Programming Interfaces (APIs) in the network as well as on the end systems pave the way for a distributed realization of Service Delivery Platforms. IT and Internet technologies represent today the foundation for programming services and implementing service platforms. Telecommunication operator service Delivery is still targeting at highest reliability and availability, which is sometimes in contrast to the fast beta service rollout in the Internet. New approaches for SDPs such as mechanisms for quality of service resp. quality of experience guarantee and performance evaluation as well for cost efficient and scalable service platforms allowing for flexible service composition are needed to integrate both paradigms.

In view of the new ecosystems and the "long tail" vision of services, SDPs particularly play an important strategic role for the economy as service factories. There was a big IN research community in Germany in the nineties. Today the SDP research field seems to be fragmented and not coordinated. Thus, in order to establish again a scientific SDP community in Germany, a new discussion forum (Fachgespräch) has been founded in KuVS under the name "Next Generation Service Delivery Platforms and Service Overlay Networks". Objective of this forum is to foster the SDP community and prepare the ground for a consolidated view on the SDP related scientific research and industrial development. The topics to be covered by this forum include:

- SDP evolution and interworking,

- Service Oriented Architecture (SOA) in telecommunications, 
- Distributed (P2P) vs. network centric service platforms,

- Self-organized service overlays,

- SDP interoperability and exposure through standardization and open network APIs,

- Network abstraction,

- IP-based service signaling (beyond SIP),

- Service creation and deployment,

- Service enablers (telecom, web, and beyond),

- Service registration and discovery,

- Service brokers, service composition, service orchestration,

- SDP management (provision, operations, maintenance, charging, security),

- IT/middleware for SDPs,

- Performance evaluation,

- SDP simulators, testbeds, and toolkits, and

- SDP standardization.

Selected papers on this special issue have been presented at the kick-off meeting of the Fachgespräch, i.e. the first GI/ITG KuVS workshop on Next Generation Service Delivery Platforms and Service Overlay Networks, that took place in Berlin, Germany on November 13, 2009. Around 50 attendants from industry and academia joined this kick-off meeting and created an excellent podium for detailed discussions.

The selection of articles represents the vast diversity of the research field of Service Delivery Platforms targeting the "upper layers" of communication and information systems in terms of protocols, algorithms and APIs. The first article presents SDP concepts based on network-based server infrastructure from an industry viewpoint. The second and third articles address service development and delivery in overlay networks. The composition and execution of service components is the focus of the fourth article. The last article takes a different view on the underlying network, targeting service execution challenges in wireless ad hoc networks.

In their article "ISA - Towards an Integrative Service Architecture for Telcos," E. Steffens, J. Heuer and H. Arnold represent the telecommunication operator viewpoint. The authors explain how an integration of telecom and web concepts would lead to a next generation SDP. The web community provides innovative and popular services that are open and can enrich telecom service offerings. The strengths of today's telecom platforms are to support the management of digital assets across networks and devices with superior user experience. Major design principles for ISA include the establishment of an enabler sphere, user centricity and bidirectional service integration.

In contrast to centralized service control systems, which incur high maintenance effort, decentralization can support higher scalability and cost efficiency through self organization and a better utilization of the infrastructure. The next two articles focus on service overlay networks supporting service development and service delivery based on a distributed architecture deployed on end systems.

In the second article C. Hübsch, C. Mayer, and O. Waldhorst present in their article "The Ariba Framework for Application Development using Service Overlays" a framework facilitating the development of end-system based decentralized services in the Internet through self-organizing service overlays. Their concept based on node and link IDs provides a level of indirection abstracting from current network obstacles such as middleboxes and mobility for service provisioning.

In the third article, which addresses distributed services entitled "Towards Measurement Consolidation for Overlay Optimization and Service Placement", D. Haage, and R. Holz present UNISONO, a generic framework for distributed network measurements. Distributed services and overlay networks rely on accurate information about the current state of nodes and the network to increase performance, efficiency and user experience. UNISONO provides such information while hiding the complexity of the measurement task. UNISONO supports consolidated measurements for the creation of overlays as well as for service placement.

In the fourth article of this special issue, N. Blum, and T. Margaria present "An Open Service Environment for Service Exposure and Orchestration of Heterogeneous NGN Services". This article discusses the design of a middleware architecture based on Service Oriented Architecture (SOA) principles. In particular the authors focus on a service broker allowing the exposure of APIs and their integration into service creation environments while considering constraints during service composition and execution.

With the last article, a shift in the underlying network infrastructure is taken: ad hoc networks are regarded as a special kind of mobile network infrastructure characterized by mobile devices communicating directly, i.e., without base stations, over wireless links with each other. In particular, G. Wittenburg, and J. Schiller focus in their article "Service Placement in Ad Hoc Networks" on the challenge of application service placement.

Finally we would like to thank all members of Fachgespräch and in particular the authors of these special issue papers. New members are very welcome to next meetings already scheduled for May and October 2010. For more information on the Fachgespräch, please contact the editors, visit the KuVS [1] or the Fachgespräch [2] web page or subscribe to the mailing list [3].

\section{References}

[1] http://www.kuvs.de/fachgespr/service-delivery-platformsngsdp/

[2] http://www.fokus.fraunhofer.de/go/kuvs_sdp_fg

[3] Mailing List: sdp_group@ fokus.fraunhofer.de 